\title{
Fast, Robust DC And Transient Fault Simulation For Nonlinear Analogue Circuits
}

\author{
Z.R. Yang and M.Zwolinski \\ Department of Electronics and Computer Science \\ University of Southampton \\ Southampton SO17 1BJ \\ mz@ecs.soton.ac.uk
}

\begin{abstract}
The evaluation of testing and design for test strategies for analogue and mixed-signal circuits requires efficient analogue fault simulation. By analogy with digital fault simulation, concurrent analogue fault simulation has been proposed to reduce simulation times by avoiding repeated construction of the circuit matrix. Simulation efficiency can be improved by dropping non-convergent faults and by fault collapsing. A robust, fast algorithm for concurrent analogue fault simulation is presented in this paper. Three techniques for automatic fault collapsing and dropping are addressed: a robust closeness measurement technique; a late start rule and an early stop rule. The algorithm has been successfully applied to both DC and transient analyses. A significant increase in the speed of analogue fault simulation has been obtained.
\end{abstract}

\section{Introduction}

Fault simulation of analogue integrated circuits has been of considerable interest in recent years. Fault modelling and simulation with SPICE has been necessary for the evaluation of design for test strategies. Normally, all possible faults have to be simulated, which is very time-consuming, particularly when parametric variations are taken into account. Hence efforts have been made to reduce the size of the fault lists and to use macro modelling. Some recent work has concentrated on speeding up the simulation algorithms themselves, and it is with this topic that this paper is concerned.

In [1] it was proposed that analogue fault simulation could be speeded up using techniques analogous to those used in concurrent digital fault simulation. The fault-free and faulty simulations would be performed concurrently in a single simulation run. Suppose that all or part of a faulty version of a circuit were to perform in the same way as the fault-free version. By checking the terminal voltages of semiconductor devices in both the faulty and fault-free versions of the circuit at each Newton-Raphson iteration at each time point in a transient analysis, redundant evaluations of device equations could be avoided. The evaluation of semiconductor device equations may take $60 \%$ or more of the simulation time, hence significant speed improvements could, in principle, be obtained.

The work reported in [3] partitioned the circuit matrix into two parts, a constant part and a variable part. The variable part is normally much smaller than the constant. The simulation time can therefore be reduced if the simulation time spent in circuit matrix construction could be significantly reduced.

In [5] the faults were ordered at each simulation iteration so that the initial guess for the fault simulation can be well defined and the total fault simulation iterations can be reduced to a significant level.

The work reported in [4] aimed to reduce fault simulation time by fault collapsing and resulted in a large reduction of fault simulation time.

Concurrent fault simulation for analogue circuits has been considered in [1], [6]. For efficient simulation it is necessary to know where and when to automatically drop faults from the fault list. The key to fault collapsing for analogue circuit simulation is to measure the closeness between the fault-free circuit and the faulty circuits. As will be discussed in this paper, a single-point closeness measurement [5] is not reliable. A robust fault collapsing technique is therefore proposed based on a multi-point closeness measurement. In general, the earlier the fault collapsing, the better the algorithm. However, there is always an unstable stage in simulating analogue circuits, in particular, during DC analysis, because of the iterative methods employed. At a very early stage of the simulation, a circuit's state can be misleading. Hence, the selection and determination of a suitable point for taking the closeness measurement is very important. We call this a late start rule. We also know that different fault simulations converge at different rates in DC analysis. Applying the same stop rule to all the fault simulations would inevitably waste resources. It is, therefore, necessary to apply an early stop rule to fault simulations. 
A robust and fast concurrent fault simulator has been implemented. This paper is organised as follows. The next section has a discussion of the closeness measurement. The third section gives the algorithm for concurrent fault simulation. The fourth section has some examples.

\section{Closeness measurement}

\subsection{Single-point measurement}

In order to reduce the computational effort needed for fault simulations, similarities between simulations of faulty circuits and of the fault-free circuit have to be identified. To do this, a measure of closeness is needed. For two vectors of real numbers, the closeness can be measured by an absolute distance measurement [2], such as the Euclidean distance, the Hamming distance or the Manhatten distance.

The Euclidean distance is the most common method for measuring the distance and was the approach adopted in [6], with very limited success. The Hamming distance is useful for measuring the distance between vectors which only contain binary numbers. The Manhatten distance is measured between two pattern swarms.

\subsection{Multi-point measurement}

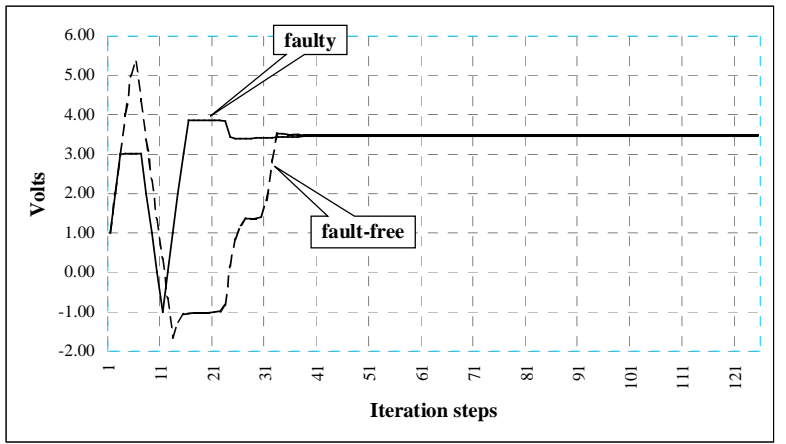

Figure 1. An illustration of non-constant distance between a faulty circuit and the fault-free circuit.

All these distance calculation methods use two single points (Euclidean and Hamming distance) or pattern swarms (Manhatten distance). It is doubtful that they can accurately highlight the closeness between faulty circuits and the fault-free circuit because the distance will not be always the same during a simulation. Figure 1 illustrates this. The vertical axis indicates the response (volts) and the horizontal axis denotes the iteration steps. The solid line shows the response of a faulty circuit and the dotted line that of the fault-free circuit. It can be seen that the distance between the faulty circuit and the fault-free circuit is not the same between the $1^{\text {st }}$ and the $40^{\text {th }}$ iterations, although at several iterations the two curves coincide. Moreover, the faulty circuit and the fault-free circuit behave identically for a few iterations. Therefore we replace the single-point closeness measurement by a multi-point closeness measurement, which is conducted during $M$ iterations:

$$
\hat{d}_{f}^{E}=\frac{1}{M} \sum_{m=1}^{M}\left\|\mathbf{x}_{0}^{m}-\mathbf{x}_{f}^{m}\right\|,
$$

where $\mathbf{x}_{0}^{m}$ and $\mathbf{x}_{f}^{m}$ are the responses at the $m^{\text {th }}$ NewtonRaphson iteration for the fault-free circuit and the $f^{\text {th }}$ faulty circuit respectively. $M$ is the user-defined continuous steps for closeness measurement and $\hat{d}_{f}^{E}$ is the closeness measurement.

\subsection{The late start rule}

In order to obtain convergence, particularly in DC analysis, the Newton-Raphson algorithm is commonly damped. This damping may be linear or non-linear. For example, if $x^{m}$ is the value of a node voltage at the $m^{\text {th }}$ Newton-Raphson iteration, $\Delta x^{m}$ is the calculated increment and $\alpha$ is the largest change voltage allowed, e.g. 1 volt, the new value of the node voltage, $x^{m+1}$, is incremented by the smaller of $\left|\Delta x^{m}\right|, \alpha$.

Using damped values for the closeness measurement is misleading. Figure 2 shows a typical response curve. It can be seen that after 13 iterations, the response starts to change more gradually. We define this point $\left(13^{\text {th }}\right.$ iteration) as a stable point because the response at the node will not subsequently dramatically change.

Unfortunately, not all the responses at the nodes of a circuit will simultaneously arrive at their stable points. Because the change before a stable point is not a real change, using these damped results will result in misleading measurements. We therefore start the closeness measurement after most nodes in a circuit arrive at their stable points. This is the late start rule.

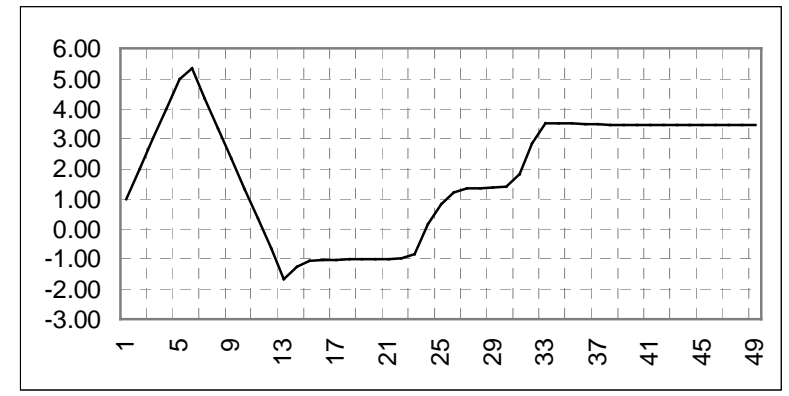

Figure 2. Illustration of a stable point. At the early stages of simulation, the change of node voltage has been damped to 1 volt. 


\section{Concurrent fault simulation using the robust fault collapsing method}

\subsection{The early stop rule}

During concurrent analogue fault simulation, in particular, during DC analysis, the fault simulations do not all converge at the same iteration step. Employing the same stop rule for all the fault simulations during concurrent simulation will hide the advantages of concurrent fault simulation. The early stop rule proposed here is very simple in that whenever one fault simulation converges, that fault simulation stops while other fault simulations carry on. Convergence of one fault simulation is measured in the usual way [7] by

$$
\widetilde{\varepsilon}=\frac{\left|x^{m+1}-x^{m}\right|}{\alpha \cdot \max \left(x^{m+1}, x^{m}\right)+\eta}
$$

where, $\alpha$ and $\eta$ are two user-defined coefficients, $x^{m}$ is a solution of a nonlinear equation at the $m^{\text {th }}$ iteration and $x^{m+1}$ is a solution of a nonlinear equation at the $(m+1)^{\text {th }}$ iteration. When $\widetilde{\varepsilon}<1$, the simulation has converged.

\subsection{Concurrent fault simulation algorithm}

From the above, we have built a robust fault collapsing method for fast and efficient concurrent analogue fault simulation. Let $\mathbf{G}_{f}$ represent the circuit description for the $f^{\text {th }}$ fault, and $\Omega^{m}$, the fault list at the $m^{\text {th }}$ iteration. A fault, $\mathbf{G}_{f} \in \Omega^{m}$, can be dropped from the fault list

$$
\Omega^{m+1}=\Omega^{m}-\mathbf{G}_{f}
$$

if $\mathbf{G}_{f}$ behaves "similarly" to the fault-free circuit,

$$
\hat{d}_{f}^{E}=\frac{1}{M} \sum_{p=m-M-1}^{m}\left\|\mathbf{x}_{0}^{p}-\mathbf{x}_{f}^{p}\right\|<T,
$$

where $T$ is the threshold for the minimum distance.

The algorithm for concurrent analogue fault simulation has been embedded into our own analogue circuit simulator. The sequence of actions for DC analysis or at one time point in transient analysis is as follows:

Step 1. Constitute the original fault list, $\Omega^{0}=\left\{\mathbf{G}^{1}, \mathbf{G}^{2}, \cdots, \mathbf{G}^{N}\right\}$, by inserting all possible $(N)$ faults into copies of the circuit. We define $\mathbf{x}_{0}^{m}, m \in[0, \infty)$ as the response vector for the fault-free circuit and $\mathbf{x}_{f}^{m}, m \in[0, \infty)$ as the response vector of the $f^{\text {th }}$ faulty circuit at the $m^{\text {th }}$ iteration during the fault simulation.

Step 2. Pre-simulate for $M$ Newton-Raphson iterations until most nodes arrive at their stable points.
Step 3. Conduct the $m^{\text {th }}$ iteration of the fault simulation for all the faulty circuits, $\mathbf{G}_{f}^{m} \in \Omega^{m-1}$, as well as for the fault-free circuit $(m>M)$. After the simulation, $\mathbf{x}_{0}^{m}$ and $\mathbf{x}_{f}^{m} \mid \mathbf{G}_{f}^{m} \in \Omega^{m-1}$ are available.

Step 4. Carry out a multi-point closeness measurement for all the faulty circuits, which are still in the fault list, $\Omega^{m-1}$. A new fault list is generated by

where

$$
\Omega^{m}=\Omega^{m-1}-\tilde{\Omega}^{m},
$$

$$
\tilde{\Omega}^{m}=\left\{\mathbf{G}_{f} \mid \hat{d}_{f}^{E}=\frac{1}{M} \sum_{p=m-M-1}^{m}\left\|\mathbf{x}_{0}^{p}-\mathbf{x}_{f}^{p}\right\|<T\right\}
$$

is the set of all the "close" faults, that have to be dropped from the fault list.

Step 5. Apply the early stop rule to $\mathbf{G}_{f}^{m} \in \Omega^{m}$. If $\widetilde{\mathcal{\varepsilon}}^{f}<1$, the $f^{\text {th }}$ fault simulation has converged. Hence $\Omega^{m}=\Omega^{m}-\mathbf{G}_{f}^{m}$.

Step 6. If one or more of the fault simulations has not converged, go back to step 3 , otherwise, stop.

\section{Examples}

Three example circuits were simulated using DC and transient to evaluate:

- whether concurrent fault simulation is faster than separate fault simulations;

- whether fault collapsing can further save fault simulation time; and

- whether the algorithm is reliable.

The first two points can be assessed by comparing the CPU time and the last can be assessed by whether the early dropping of faults retains the accuracy.

The example circuits used were a two-stage bipolar amplifier, composed of six bipolar transistors and ten resistors; a differential amplifier, composed of four bipolar transistors and five resistors; and a CMOS twostage amplifier with nine transistors.

\subsection{DC analysis}

We inserted 36 faults into the two-stage BJT amplifier. The DC fault simulations for three faults did not converge. Of the remaining 33 faults, there were 15 short faults, which are all the possible short-circuits across the terminals of the six transistors, nine shortcircuits across the resistors and nine parametric faults in the resistors (reducing the resistance values by $50 \%$ ). We conducted separate fault simulations and a concurrent 
fault simulation. In the separate fault simulations, the faulty and the fault-free circuits simulated one by one. The times of the separate fault simulations are listed in Table 1.

Table 1. The time behaviour of separate fault simulations for BJT two-stage amplifier

\begin{tabular}{|c|c|c|c|}
\hline no. & type & iterations & cpu(ms) \\
\hline 0 & Fault-free & 43 & 70 \\
\hline 1 & short & 26 & 30 \\
\hline 2 & short & 44 & 70 \\
\hline 3 & short & 31 & 60 \\
\hline 4 & short & 27 & 40 \\
\hline 5 & short & 26 & 40 \\
\hline 6 & short & 24 & 40 \\
\hline 7 & short & 36 & 40 \\
\hline 8 & short & 31 & 50 \\
\hline 9 & short & 51 & 80 \\
\hline 10 & short & 44 & 70 \\
\hline 11 & short & 41 & 70 \\
\hline 12 & short & 30 & 50 \\
\hline 13 & short & - & - \\
\hline 14 & short & 55 & 90 \\
\hline 15 & short & 23 & 40 \\
\hline 16 & short & 24 & 30 \\
\hline 17 & short & 33 & 50 \\
\hline 18 & short & 32 & 60 \\
\hline 19 & short & 43 & 70 \\
\hline 20 & short & 38 & 40 \\
\hline 21 & short & 116 & 150 \\
\hline 22 & short & 55 & 90 \\
\hline 23 & short & - & - \\
\hline 24 & short & 31 & 60 \\
\hline 25 & short & 49 & 80 \\
\hline 26 & short & 46 & 70 \\
\hline 27 & parameter & 47 & 70 \\
\hline 28 & parameter & 44 & 70 \\
\hline 29 & parameter & 43 & 60 \\
\hline 30 & parameter & 34 & 50 \\
\hline 31 & parameter & 125 & 170 \\
\hline 32 & parameter & 43 & 60 \\
\hline 33 & parameter & 50 & 80 \\
\hline 34 & parameter & 39 & 60 \\
\hline 35 & parameter & - & - \\
\hline 36 & parameter & 43 & 70 \\
\hline Total & & & 2230 \\
\hline
\end{tabular}

It can be seen that the separate fault simulations need $2230 \mathrm{~ms}$ in total. In contrast only $1810 \mathrm{~ms}$ are required by the concurrent fault simulation giving a $19 \%$ reduction in the CPU time.
If the early stop rule is not used, the concurrent simulation needs $3350 \mathrm{~ms}$ (50\% increase in CPU time). This is because a few fault simulations require more than 100 Newton-Raphson iterations, as shown in Table 1. Most other fault simulations converge in fewer than 50 iterations, but without the early stop rule have to be iterated to the limit.

If fault collapsing is applied with the threshold value set to 0.1 volt and using a 3-point measurement can further reduce the CPU time to $1590 \mathrm{~ms}$ or a further $12 \%$ giving a total of $29 \%$ saving in CPU time compared with separate fault simulation.

Similarly, we inserted 19 faults into the differential BJT amplifer and 24 faults into the CMOS opamp. One fault in the CMOS opamp simulations failed to converge. Again we performed separate fault simulations and a concurrent fault simulation. The CPU times required are summarised in Table 2.

Table 2 CPU times in $\mathrm{ms}$ for DC fault simulation of example circuits.

\begin{tabular}{|l|l|l|l|l|l||}
\hline \hline Circuit & Separate & $\begin{array}{l}\text { Concurr- } \\
\text { ent }\end{array}$ & $\begin{array}{l}\text { Early } \\
\text { Stop }\end{array}$ & $\begin{array}{l}\text { Collaps- } \\
\text { ing }\end{array}$ & Saving \\
\hline $\begin{array}{l}\text { 2-stage } \\
\text { BJT }\end{array}$ & 2230 & 3350 & 1810 & 1590 & $29 \%$ \\
\hline Diff BJT & 160 & 160 & 80 & 70 & $56 \%$ \\
\hline CMOS & 3270 & 3830 & 3200 & 2750 & $16 \%$ \\
\hline
\end{tabular}

Table 3. Fault Simulation results for DC analysis of BJT two-stage amplifier

\begin{tabular}{|c|c|c|c|c|c|}
\hline no. & $\bar{\varepsilon}^{f}$ & $\begin{array}{l}\text { collapse } \\
\text { iteration }\end{array}$ & no. & $\overline{\mathcal{E}^{f}}$ & $\begin{array}{l}\text { collapse } \\
\text { iteration }\end{array}$ \\
\hline 1 & 0.377 & 37 & 19 & 0.000 & 35 \\
\hline 2 & 0.211 & 35 & 20 & 0.829 & \\
\hline 3 & 1.217 & & 21 & 0.490 & \\
\hline 4 & 1.322 & & 22 & 0.000 & 22 \\
\hline 5 & 1.278 & & 23 & - & \\
\hline 6 & 0.028 & 35 & 24 & 1.017 & \\
\hline 7 & 0.540 & & 25 & 1.984 & \\
\hline 8 & 0.751 & & 26 & 2.031 & \\
\hline 9 & 0.363 & 35 & 27 & 0.518 & 43 \\
\hline 10 & 0.007 & 35 & 28 & 0.126 & 38 \\
\hline 11 & 0.144 & 22 & 29 & 0.000 & 35 \\
\hline 12 & 0.883 & & 30 & 0.302 & 36 \\
\hline 13 & - & & 31 & 0.024 & 122 \\
\hline 14 & 2.783 & & 32 & 0.000 & 22 \\
\hline 15 & 1.008 & & 33 & 0.664 & 22 \\
\hline 16 & 0.793 & & 34 & 0.470 & \\
\hline 17 & 1.288 & & 35 & - & \\
\hline 18 & 0.744 & & 36 & 0.411 & 22 \\
\hline
\end{tabular}


This shows that concurrent fault simulation is faster than separate fault simulation. provided that the early stop rule and fault collapsing are applied.

For DC analysis, the accuracy of fault collapsing is measured by the difference between the fault-free circuit and faulty circuits on the final results

$$
\varepsilon=\frac{1}{N} \sum_{i=1}^{N} \varepsilon_{i}^{f}=\frac{1}{N} \sum_{i=1}^{N}\left\|\mathbf{x}^{0}-\mathbf{x}_{i}^{f}\right\| .
$$

where, $N$ is the number of the faults that have been collapsed. Table 3 lists the results for the BJT two-stage amplifier. The second column is the difference between the fault-free circuit and faulty circuits. The final column gives the iteration, at which the fault is collapsed. The final error is 0.198 , which is larger than the threshold (0.1), but is still small.

\subsection{Transient analysis}

The same circuits were fault simulated in transient analyses. Convergence could not be reached for certain faults. Table 4 summarises the CPU times required for convergent faults.

Table 4 CPU times in seconds for transient fault simulation of example circuits.

\begin{tabular}{|l|l|l|l|l|l||}
\hline Circuit & Separate & $\begin{array}{l}\text { Concurr- } \\
\text { ent }\end{array}$ & $\begin{array}{l}\text { Collaps- } \\
\text { ing }\end{array}$ & Saving & $\begin{array}{l}\text { No. } \\
\text { faults } \\
\text { collapsed }\end{array}$ \\
\hline $\begin{array}{l}\text { 2-stage } \\
\text { BJT }\end{array}$ & 571 & 490 & 305 & $46 \%$ & 15 \\
\hline Diff BJT & 185 & 168 & 107 & $42 \%$ & 8 \\
\hline CMOS & 2655 & 2000 & 1041 & $61 \%$ & 9 \\
\hline
\end{tabular}

Table 5. Fault simulation results for transient analysis of BJT differential amplifier

\begin{tabular}{||l|l|l||l|l|l||}
\hline \hline no. & $\varepsilon$ & $\begin{array}{l}\text { collapse } \\
\text { time }\end{array}$ & no & $\varepsilon$ & $\begin{array}{l}\text { collapse } \\
\text { time }\end{array}$ \\
\hline 1 & 2.404 & & 11 & 1.311 & \\
\hline 2 & - & & 12 & 0.389 & $1.1340 \mathrm{e}-07$ \\
\hline 3 & - & & 13 & 0.035 & $5.5000 \mathrm{e}-10$ \\
\hline 4 & 2.200 & & 14 & 9.430 & \\
\hline 5 & - & & 15 & 0.538 & $1.1535 \mathrm{e}-08$ \\
\hline 6 & - & & 16 & 0.656 & $4.1250 \mathrm{e}-08$ \\
\hline 7 & 9.430 & & 17 & 0.199 & $1.0510 \mathrm{e}-07$ \\
\hline 8 & 0.936 & $1.0510 \mathrm{e}-07$ & 18 & 0.018 & $5.5000 \mathrm{e}-10$ \\
\hline 9 & 2.078 & & 19 & 1.707 & \\
\hline 10 & 0.075 & $1.2480 \mathrm{e}-08$ & \multicolumn{3}{|l||}{} \\
\hline \hline
\end{tabular}

Concurrent fault simulation is faster than separate fault simulations when fault collapsing is used. For transient analysis, the accuracy of fault collapsing is compared using the difference between the fault-free circuit and faulty circuits on the whole range of analysis

$$
\varepsilon^{f}=\frac{1}{T} \sum_{t=1}^{T}\left\|\mathbf{x}_{t}^{0}-\mathbf{x}_{t}^{f}\right\| .
$$

Table 5 lists the results for the differential amplifier simulated for $100 \mu \mathrm{s}$. The second column shows the greatest instantaneous difference between the faulty and fault-free circuits over the whole simulation period. The third column gives the time at which fault collapsing occurs. It can be seen that in the worst case, the collapsed faults differ from the fault-free circuit behaviour by less than a volt.

\section{Summary and further work}

A robust, fast concurrent analogue fault simulation algorithm has been proposed and verified in this paper. For DC analysis, a 56\% reduction in CPU time can be achieved, while $61 \%$ of CPU time can be saved for the case of transient analysis. It should be noted that this achievement depends on circuit types on which different circuits have different degree of fault diversity. In the sense of fault collapse, we should emphases on what we can do rather than what the average achievement. The algorithm has been realised in C on a SUN UltraSPARC under Solaris. The implementation of this algorithm is simple and the whole algorithm has been embedded in a circuit simulator. The most significant difference of this algorithm to traditional analogue fault simulation is that fault collapsing has been realised as an automatic mechanism embedded into the circuit simulator.

The example circuits used have been small and have not contained hierarchy. It is expected that larger, hierarchical circuits will exhibit more significant savings.

Further work will focus on how to embed the clustering technique to realise comprehensive fault collapsing and how to automatically drop non-converging faulty circuits from the fault list during a concurrent fault simulation.

\section{Acknowledgments}

This work has been supported by EPSRC grant GR/L35829.

\section{References}

1. M.Zwolinski, "Relaxation Methods for Analogue Fault Simulation", 20th Int. Conf. Microelectronics (MIEL'95), Niš, Serbia, pp. 467-471, 1995.

2. G. B. Bruce, Pattern Recognition, Ideas in Pratice, Plenum Press, 1978.

3. J. Hou and A. Chatterjee, "CONCERT: a concurrent fault simulator for analog circuits", $4^{\text {th }}$ IEEE International MixedSignal Testing Workshop, June $8^{\text {th }}-11^{\text {th }}, 1998$, Atlantic Hotel, The Hague, The Netherlands, 1998.

4. A. J. Perkins, M. Zwolinski, C. D. Chalk, and B. R. Wilkins, "Fault modeling and simulation using VHDL-AMS", Analog Integrated Circuits and Signal Processing, vol. 16, no. 2, pp. 141-156, 1998. 
5. C. J. Shi and M. W. Tian, "Efficient DC fault simulation of nonlinear analog circuits", Design, Automation and Test in Europe Conference (DATE '98), Paris, France, Feb. 23-26, 1998.

6. M. Zwolinski, A. D. Brown and C. D. Chalk, "Concurrent analogue fault simulation", pp. 42-47, in Proc. $3^{\text {rd }}$ IEEE International Mixed-Signal Testing Workshop, 1997.

7. J. Vlach and K. Singhal, Computer Methods for Circuit Analysis and Design, Van Nostrand Reinhold, New York, 1983. 